\title{
Hypsochromic solvent shift of the charge separation band in ionic donor-acceptor $\mathrm{Li}^{+} @ \mathrm{C}_{60} \subset[10] \mathrm{CPP}$
}

\author{
Anton J. Stasyuk, ${ }^{[a]^{*}}$ Olga A. Stasyuk, ${ }^{[a]}$ Miquel Sola, ${ }^{[a]^{*}}$ and Alexander A. Voityuk ${ }^{[a, b]^{*}}$
}

\begin{abstract}
Donor-acceptor dyads with $\mathrm{C}_{60}$ as electron acceptor and a cycloparaphenylene (CPP) unit assembled via non-covalent interactions as electron donor have been reported in the literature. In this work, we study computationally using the DFT/TDDFT approach the photoinduced electron transfer (PET) in CPP-based donoracceptor supramolecule $\mathrm{C}_{60} \subset[10] \mathrm{CPP}$ and $\mathrm{Li}^{+} @ \mathrm{C}_{60} \subset[10] \mathrm{CPP}$. As it is well known, the energy of charge separated (CS) states generated in donor-acceptor systems by PET are significantly stabilized by polar environment. Based on the analysis of initial and final states after PET in various fullerene complexes, we find a system, $\mathrm{Li}^{+} @ \mathrm{C}_{60} \subset[10] \mathrm{CPP}$, that shows anomalous solvent effects, i.e., destabilization of charge separated states by polar medium. To our knowledge, this is the first example of fullerene based systems where a hypsochromic shift of the CS band is demonstrated. The CS reaction in the $\mathrm{Li}^{+} @ \mathrm{C}_{60} \subset[10] \mathrm{CPP}$ complex is found to occur in the inverted Marcus regime. In spite of that the CS reaction rate becomes faster with increasing solvent polarity of the environment.
\end{abstract}

Conversion of sunlight into more accessible forms of energy, such as electrical or chemical ones, is a one of primary challenge for the human race. A lot of attention and efforts have been paid to design and prepare model compounds that mimics natural photosynthetic systems. ${ }^{[1]}$ Generation of a long-lived chargeseparated (CS) state with high quantum yield and separation of radical ion pairs over long distances to prevent their recombination are extremely important conditions for photosynthetic systems. ${ }^{[2]}$ Most of photosynthetic reactions, both natural and artificial, take place in solution or media that significantly influences this process. ${ }^{[3]}$ Multi-component systems mimicking natural photosynthetic processes have been designed and extensively studied. Among potentially suitable chromophores, much attention has been paid to boron dipyrromethane (BODIPY) and its derivatives, ${ }^{[4]}$ porphyrinoids ${ }^{[1 a}$, 5] and other rigid and planar structures with a high extinction coefficient and fluorescence quantum yield. As an acceptor unit, one uses often fullerenes which demonstrate a strong electron

[a] Dr. A. J. Stasyuk, Dr. O. A. Stasyuk, Prof. Dr. M. Solà, Prof. Dr. A. A. Voityuk

Institute of Computational Chemistry and Catalysis and Department of Chemistry

University of Girona

C/ M. Aurèlia Capmany, 69, 17003 Girona, Spain.

E-mail: antony.stasuk@gmail.com

E-mail: miquel.sola@udg.edu

E-mail: alexander.voityuk@icrea.cat

[b] Prof. Dr. A. A. Voityuk

Institució Catalana de Recerca i Estudis Avancats (ICREA), 08010 Barcelona, Spain

Supporting information for this article is given via a link at the end of the document. withdrawing power, high electron delocalization ability, and small reorganization energy. ${ }^{[6]}$

Electronic communication between donor (D) and acceptor $(A)$ is a key feature in the design of photosystems. Electronic and structural nature of bridging spacer between donor and acceptor can dramatically influence the photoinduced electron transfer (PET) dynamics. ${ }^{[7]}$ D-A systems assembled via non-covalent interactions are expected to give rise to efficient PET, thus representing an attractive alternative to covalently linked analogs. ${ }^{[8]}$ The dispersion interaction is responsible for stabilization of such complexes. ${ }^{[9]}$ Because of that, the use of concave-convex complementarity is an excellent strategy to increase the stability of the fullerene complexes. ${ }^{[8 \mathrm{~d}, 10]}$ Cycloparaphenylenes (CPPs) are hoop-shaped m-conjugated molecules in which paraphenylene units form a cycle. CPPs have attracted the attention of scientists due to their interesting properties arising from distorted and strained aromatic system and radially oriented $\pi$-orbitals. ${ }^{[11]}$ Nanosized concave cavities of CPPs, so-called carbon nano-rings, can act as hosts for $\pi$ conjugated molecules with convex surfaces, such as fullerenes. ${ }^{[12]}$

In 2011, Yamago and co-workers ${ }^{[12 d]}$ demonstrated that $\mathrm{C}_{60}$ can be selectively encapsulated by [10]CPP forming stable $\mathrm{C}_{60} \subset[10] C P P$ complex in toluene with a binding constant $\left(K_{a}\right)$ of $2.79 \pm 0.03 \times 10^{6} \mathrm{~mol} / \mathrm{L}$. Later, Itami and co-workers ${ }^{[13]}$ successfully synthesized and characterized $\mathrm{Li}^{+} @ \mathrm{C}_{60} \subset[10] \mathrm{CPP}$ complex with $K_{a}=(4.78 \pm 0.66) \times 10^{4} \mathrm{~mol} / \mathrm{L}$ in dichloromethane. Electrochemical measurements and spectroscopic analysis revealed strong charge-transfer interaction between [10]CPP and $\mathrm{Li}^{+} @ \mathrm{C}_{60}$ parts. Herein we report a comprehensive analysis of photoinduced charge separated states in $\mathrm{C}_{60} \subset[10] \mathrm{CPP}$ and $\mathrm{Li}^{+} @ \mathrm{C}_{60} \subset[10] \mathrm{CPP}$ complexes using Time-Dependent DFT theory coupled with conductor-like polarizable continuum model (CPCM) to account for environmental effects. Lowest-lying CS states of $\mathrm{Li}^{+} @ \mathrm{C}_{60} \subset[10] \mathrm{CPP}$ complex demonstrate unique behavior towards solvation. They are strongly destabilized by the solvent. Moreover, destabilization effect increases with increasing polarity of the solvent.

Stability and ground state properties. Geometries of $\mathrm{C}_{60} \subset[10] \mathrm{CPP}$ and $\mathrm{Li}^{+} @ \mathrm{C}_{60} \subset[10] \mathrm{CPP}$ (Figure 1) were optimized using BLYP-D3(BJ)/def2-SVP functional. ${ }^{[14]}$ Subsequent excited state calculations were performed using time-dependent DFT formalism with range-separated CAM-B3LYP ${ }^{[15]}$ functional (see full computational details in the $\mathrm{SI}$ ). For the $\mathrm{C}_{60} \subset[10] \mathrm{CPP}$ system, the interaction energy is found to be $-53.8 \mathrm{kcal} / \mathrm{mol}$, in a good agreement with previously estimated values by RodríguezOtero $^{[16]}$ and Zhao ${ }^{[17]}$ using other DFT functionals. It is worth mentioning that doping of this system by $\mathrm{Li}^{+}$leads to noticeable stabilization of complex associated with superadditive effect similar to that found for carbon nano-onions (CNOs). ${ }^{[18]}$ The stabilization energy of $\mathrm{Li}^{+} @ \mathrm{C}_{60} \subset[10] \mathrm{CPP}$ complex is -94.8 
$\mathrm{kcal} / \mathrm{mol}$, which is almost $11 \mathrm{kcal} / \mathrm{mol}$ higher than sum of energies of the individual species, i.e. $\mathrm{Li}^{+} @ \mathrm{C}_{60}$ and $\mathrm{C}_{60} \subset[10] \mathrm{CPP}$ (Table S1).

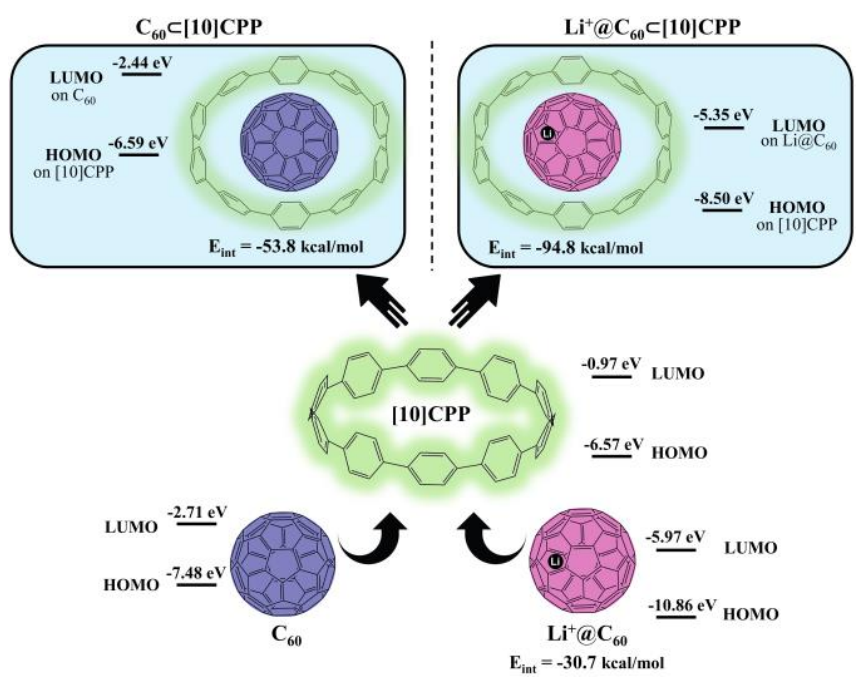

Figure 1. HOMO and LUMO energies, and their localization in $\mathrm{C}_{60} \subset[10] \mathrm{CPP}$, $\mathrm{Li}^{+} @ \mathrm{C}_{60} \subset[10] \mathrm{CPP}$ systems and corresponding individual fragments ([10]CPP, $\mathrm{Li}^{+} @ \mathrm{C}_{60}$, and $\mathrm{C}_{60}$ ).

As can be seen from Figure 1, LUMO in the $\mathrm{Li}^{+}$-doped $\mathrm{C}_{60}$ is by $3.26 \mathrm{eV}$ lower than in the original $\mathrm{C}_{60}$ mainly due to electrostatic potential of $\mathrm{Li}^{+}$. In final complexes, electron-withdrawing abilities of pristine and $\mathrm{Li}^{+}$-doped fullerenes change only slightly compared to their free state. At the same time, the effect of association is more pronounced for the energy of the HOMO localized on [10]CPP fragment. The energy of the HOMO does not change when complex with $\mathrm{C}_{60}$ is formed, whereas $\mathrm{Li}^{+} @ \mathrm{C}_{60}$ counterpart lowers the HOMO energy of [10]CPP by nearly $2 \mathrm{eV}$. Overall, HOMO-LUMO gap in $\mathrm{Li}^{+} @ \mathrm{C}_{60} \subset[10] \mathrm{CPP}$ complex is $1 \mathrm{eV}$ smaller than in $\mathrm{C}_{60} \subset[10] \mathrm{CPP}$ (3.15 and $4.15 \mathrm{eV}$, respectively). Electronic structure of the $\mathrm{C}_{60} \subset[10] \mathrm{CPP}$ complex in the ground state (GS) can be described as two neutral fragments without charge separation. In case of $\mathrm{Li}^{+}$-doped complex, most of charge is localized on $\mathrm{Li}(0.77 \mathrm{e})$, while [10]CPP and $\mathrm{C}_{60}$ units are almost neutral.

Singlet excited states. In order to characterize properties of excited states, $\mathrm{C}_{60} \subset[10] \mathrm{CPP}$ and $\mathrm{Li}^{+} @ \mathrm{C}_{60} \subset[10] \mathrm{CPP}$ systems were divided into 2 and 3 fragments, respectively (Figure S1, SI): 1)[10]CPP; 2) $\mathrm{C}_{60}$; and 3) $\mathrm{Li}^{+}$(only in case of $\mathrm{Li}^{+} @ \mathrm{C}_{60} \subset[10] \mathrm{CPP}$ ). Exciton delocalization and charge transfer contributions were analyzed for 60 lowest excited states of each complex. Three types of excited states were identified: locally excited states (LE) - exciton is mostly localized on single fragment (charge separation value $<0.1 \mathrm{e}$ ); CS states where electron density is transferred between two fragments (charge separation $>0.9 \mathrm{e}$ ); and mixed states with significant contributions of LE and CS (charge separation is between $0.1 \mathrm{e}$ and $0.9 \mathrm{e}$ ).

In the gas phase, the vertical singlet excitation energies of $\mathrm{C}_{60} \subset[10] C P P$ range from 2.50 to $4.30 \mathrm{eV}$. The LE state localized on $\mathrm{C}_{60}$ has the lowest energy and is associated with a HOMO-3 to LUMO+1 transition. LE states on [10]CPP unit are located at
$3.46 \mathrm{eV}$ and correspond to $\mathrm{HOMO}$ to $\mathrm{LUMO}+7$ transitions. The first CS state found at $2.76 \mathrm{eV}$ corresponds to electron transfer from [10]CPP to $\mathrm{C}_{60}$ (Table 1). This state can be described as purely HOMO-LUMO transition with 0.98 e transferred. Therefore, the CS structure corresponds to $\mathrm{C}_{60} \subset[10] \mathrm{CPP}^{+}$. This CS transition has a relatively weak oscillator strength of about 0.001 .

Table 1. Singlet excitation energies $(E, e V)$, the main singly excited configuration $(\mathrm{HOMO}(\mathrm{H})-\mathrm{LUMO}(\mathrm{L}))$ and its weight $(\mathrm{W})$, the oscillator strength (f), and the extent of charge separation (CS, e) or exciton localization $(\mathrm{X})$

\begin{tabular}{|c|c|c|c|c|}
\hline & \multicolumn{4}{|c|}{ Supramolecule } \\
\hline & \multicolumn{2}{|c|}{$\mathrm{C}_{60} \subset[10] \mathrm{CPP}$} & \multicolumn{2}{|c|}{$\mathrm{Li}^{+} @ \mathrm{C}_{60} \subset[10] \mathrm{CPP}$} \\
\hline & VAC & $\mathrm{DCM}$ & VAC & DCM \\
\hline & \multicolumn{4}{|c|}{ LE1 (Fullerene) } \\
\hline $\begin{array}{l}\text { E } \\
\text { Transition } \\
\text { (W) }\end{array}$ & $\begin{array}{c}2.495 \\
\mathrm{H}-3-\mathrm{L}+1 \\
(0.42) \\
<0.001\end{array}$ & $\begin{array}{c}2.489 \\
\mathrm{H}-3-\mathrm{L}+2 \\
(0.47) \\
<0.001\end{array}$ & $\begin{array}{c}2.480 \\
\mathrm{H}-6-\mathrm{L}+1 \\
(0.30) \\
<0.001\end{array}$ & $\begin{array}{c}2.524 \\
H-3-L+2 \\
(0.24) \\
<0.001\end{array}$ \\
\hline $\mathrm{X}$ & \multicolumn{4}{|c|}{ LE2 ([10]CPP) } \\
\hline $\begin{array}{l}\mathrm{E} \\
\text { Transition } \\
(\mathrm{W}) \\
\mathrm{f} \\
\mathrm{X}\end{array}$ & $\begin{array}{c}3.457 \\
H-L+7 \\
(0.34) \\
<0.001 \\
0.955\end{array}$ & $\begin{array}{c}3.462 \\
H-L+7 \\
(0.31) \\
<0.001 \\
0.953\end{array}$ & $\begin{array}{c}3.368 \\
H-L+7 \\
(0.47) \\
<0.001 \\
0.901\end{array}$ & $\begin{array}{c}3.431 \\
H-L+6 \\
(0.34) \\
<0.001 \\
0.918\end{array}$ \\
\hline $\mathrm{X}$ & \multicolumn{4}{|c|}{ Most absorbtive transition ([10]CPP) } \\
\hline $\begin{array}{l}\text { E } \\
\text { Transition } \\
\text { (W) } \\
f \\
X\end{array}$ & $\begin{array}{c}3.849 \\
H-1-L+3 \\
(0.16) \\
0.676 \\
0.652\end{array}$ & $\begin{array}{c}3.868 \\
H-1-L+7 \\
(0.26) \\
1.230 \\
0.833\end{array}$ & $\begin{array}{c}3.813 \\
\mathrm{H}-1-\mathrm{L}+11 \\
(0.13) \\
0.304 \\
0.237\end{array}$ & $\begin{array}{c}3.766 \\
\mathrm{H}-1-\mathrm{L}+10 \\
(0.27) \\
0.971 \\
0.710\end{array}$ \\
\hline & \multicolumn{4}{|c|}{ CS ([10]CPP $\rightarrow$ Fullerene) } \\
\hline $\begin{array}{l}\mathrm{E} \\
\text { Transition } \\
(\mathrm{W}) \\
\mathrm{f} \\
\mathrm{CS}\end{array}$ & $\begin{array}{l}2.762 \\
H-L \\
(0.96) \\
0.001 \\
0.983\end{array}$ & $\begin{array}{l}2.555 \\
H-L \\
(0.96) \\
0.001 \\
0.983\end{array}$ & $\begin{array}{c}1.720 \\
H-L+1 \\
(0.88) \\
<0.001 \\
0.985\end{array}$ & $\begin{array}{c}2.060 \\
H-L+1 \\
(0.79) \\
<0.001 \\
0.986\end{array}$ \\
\hline
\end{tabular}

For structurally similar $\mathrm{C}_{60} @ \mathrm{C}_{240}$ nano-onion, we have previously demonstrated that encapsulation of $\mathrm{Li}^{+}$cation leads to considerable changes in electronic structure of CNOs. ${ }^{[18 \mathrm{~b}]}$ Particularly, we observed the energy gap decreases by about 0.5 eV. The change in the HOMO-LUMO gap can be justified by electrostatic stabilization of the CS state by $\mathrm{Li}^{+}$cation, while the LE states without separated charges are almost insensitive to the ion encapsulation. Computation of singlet excited states for $\mathrm{Li}^{+} @ \mathrm{C}_{60} \subset[10] \mathrm{CPP}$ expectedly revealed that energy of the lowest excited state decreased by $0.78 \mathrm{eV}$, from 2.50 to $1.72 \mathrm{eV}$. Decrease in energy of the lowest excited state is accompanied by a change in the nature of this state. For $\mathrm{Li}^{+} @ \mathrm{C}_{60} \subset[10] \mathrm{CPP}$ system, the lowest excited state corresponds to the CS state with electron transfer from [10]CPP to $\mathrm{C}_{60}$ unit. Energies of the LE states remain almost unchanged, $2.48 \mathrm{eV}\left(\mathrm{LE}\right.$ on $\mathrm{C}_{60}$ ) and $3.37 \mathrm{eV}$ (LE on [10]CPP). Our findings are in perfect agreement with electrochemical measurements and spectroscopic observations by Itami and co-workers. ${ }^{[11]}$ Structure of the CS state can be described as $\mathrm{Li}^{+} @ \mathrm{C}_{60} \subset[10] \mathrm{CPP}^{+}$. The frontier molecular orbitals representing the LE and CS states are shown in Figure S2.

Environment effect on CS states. To evaluate the effect of solvation on the excited states, a COSMO-like model[19] was 
applied with dichloromethane (DCM) as the solvent. The GS solvation energies are -0.86 and $-1.91 \mathrm{eV}$ for $\mathrm{C}_{60} \subset[10] \mathrm{CPP}$ and $\mathrm{Li}^{+} @ \mathrm{C}_{60} \subset[10] \mathrm{CPP}$, correspondingly. The solvation energy of $\mathrm{Li}^{+}-$ doped complex is noticeably larger because system is charged. Usually for LE states, changes in the solvation energies with respect to the GS are relatively weak due to similarity in dipole moments, while for CT states the effect can be significant. ${ }^{[20]}$ However, high symmetry of the studied complexes and ability of both fragments to effectively delocalize the charge allowed us to assume that even for the CS states the difference in solvation energies compared to the ground state should not be large. Calculations showed that differences in dipole moments between GS and CS states in $\mathrm{C}_{60} \subset[10] \mathrm{CPP}$ complex are $0.4 \mathrm{D}$, whereas for LE state this difference is $0.1 \mathrm{D}$. Consequently, the solvation energy of the CS state changes from 2.76 to $2.55 \mathrm{eV}$ leading to a bathochromic shift by $36 \mathrm{~nm}$. The observed stabilization of the CS state is insufficient to reorder the CS and LE states by passing from the gas phase to DCM solution.

Our modeling of the solvation effect for $\mathrm{Li}^{+} @ \mathrm{C}_{60} \subset[10] \mathrm{CPP}$ complex revealed an interesting feature. The CS state exhibits a noticeable hypsochromic shift. The excitation energy changes from 1.72 to $2.06 \mathrm{eV}$ when going from the gas-phase to the DCM media. To get more insight into the environmental effect, we have additionally performed excited state simulations for 6 solvents of different polarity, from $\varepsilon=2$ ( $n$-hexane) to $\varepsilon=25$ (benzonitrile). The gas-phase geometry was used in all cases to avoid any effect associated with geometry changes. Calculations of the $\mathrm{C}_{60} \subset[10] \mathrm{CPP}$ complex, as a reference, were also performed. In all solvents, LE states for both $\mathrm{C}_{60} \subset[10] \mathrm{CPP}$ and $\mathrm{Li}^{+} @ \mathrm{C}_{60} \subset[10] \mathrm{CPP}$ show the solvation energies similar to the GS, providing only negligible changes in the excitation energies (detailed data provided in Table S2). In contrast, the CS transition energies depend on the solvent polarity. As can be seen in Figure 2, the lowest CS state of $\mathrm{Li}^{+}$-doped complex demonstrates a big hypsochromic shift of about $0.4 \mathrm{eV}$.
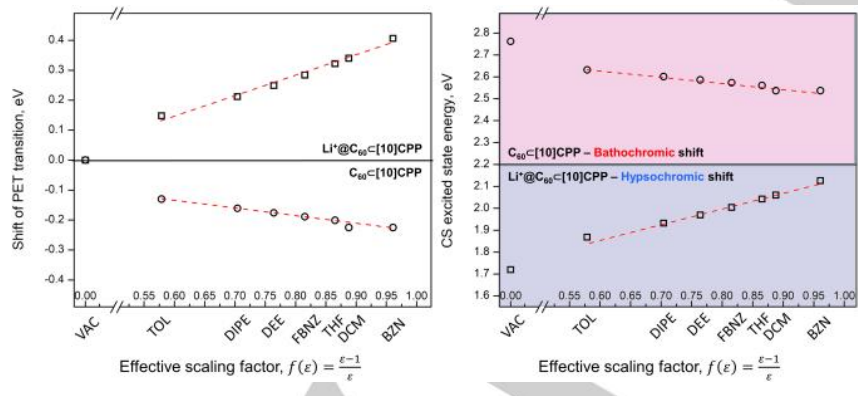

Figure 2. Left: Shift of PET transition for $\mathrm{Li}^{+} @ \mathrm{C}_{60} \subset[10] \mathrm{CPP}$ (top) and $\mathrm{C}_{60} \subset[10] C P P$ (bottom) as a function of solvent polarity. Right: Solvent dependent shifts of CS state energy for $\mathrm{C}_{60} \subset[10] \mathrm{CPP}$ (top) and $\mathrm{Li}^{+} @ \mathrm{C}_{60} \subset[10] \mathrm{CPP}$ (bottom) in toluene (TOL), diisopropyl ether (DIPE), diethyl ether (DEE), fluorobenzene (FBNZ), tetrahydrofuran (THF), dichloromethane (DCM), and benzonitrile (BZN).

The Gibbs solvation energy estimated with the PCM model is determined by two terms: electrostatic $E_{\mathrm{el}}$ and non-electrostatic $E_{\text {non }}$ energies. In turn, the last term may be divided into three parts including the dispersion, repulsion and cavitation components. ${ }^{[21]}$
Because by a vertical transition, the geometry of the system remains unchanged, the solvation energy change by a difference in $E_{\text {el }}$ of the initial and final states. To explain the hypsochromic shift of the CS transition, the molecular electrostatic potential (MEP) of $\mathrm{Li}^{+} @ \mathrm{C}_{60} \subset[10] \mathrm{CPP}$ complex in its GS and CS excited state was calculated (Figure 3).
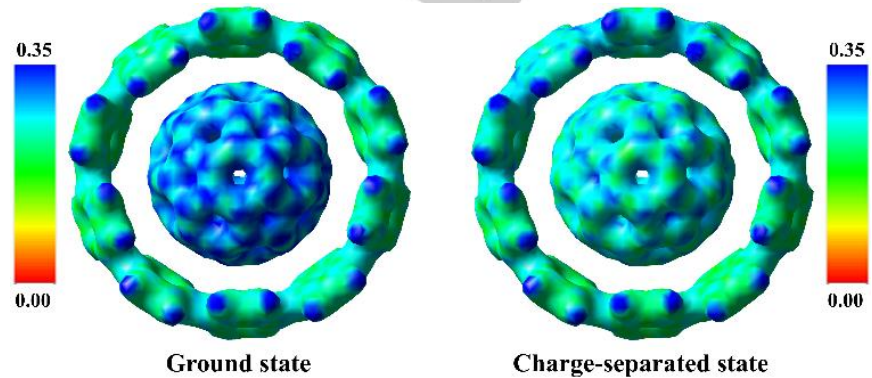

Figure 3. MEP of the ground and CS states of $\mathrm{Li}^{+} @ \mathrm{C}_{60} \subset[10] \mathrm{CPP}$ drawn on the isodensity surface of $0.05 \mathrm{e} / \AA^{3}$.

A large difference between the GS and CS MEPs is observed around the $\mathrm{C}_{60}$ unit. By PET, $\mathrm{Li}^{+} @ \mathrm{C}_{60} \subset[10] \mathrm{CPP} \rightarrow \mathrm{Li}^{+} @ \mathrm{C}_{60}$ $\subset[10] \mathrm{CPP}^{+}$, the total charge on $\mathrm{Li}^{+} @ \mathrm{C}_{60}$ becomes almost neutral decreasing the MEP in its vicinity. MEP changes around [10]CPP are less pronounced due to efficient delocalization of the positive charge over the entire unit.

A more elegant qualitative explanation for the observed phenomenon can also be provided. Let us divide the $\mathrm{Li}^{+} @ \mathrm{C}_{60} \subset[10] \mathrm{CPP}$ complex into 2 (rather than 3) parts, $\mathrm{Li}^{+} @ \mathrm{C}_{60}$ and [10]CPP. Then the charge separation between $\mathrm{C}_{60}$ and [10]CPP can formally be described as charge shift from $\mathrm{Li}^{+} @ \mathrm{C}_{60}$ to [10]CPP. For a charge-shift reaction, $D^{+} \cdots A \rightarrow D \cdots A^{+}$, a change in solvation energy is determined by the size of the $D$ and $A$ sites, providing the electrostatic potential is uniformly distributed around $\mathrm{D}^{+}$and $\mathrm{A}^{+}$). The band shift can be roughly estimated using the Born equation:

$$
\Delta h v \approx \frac{q^{2}}{2}\left(\frac{\varepsilon-1}{1}\right)\left(\frac{1}{R_{D}}-\frac{1}{R_{A}}\right)
$$

where $\varepsilon$ is the solvent dielectric constant. Because [10]CPP has a larger radius than $\mathrm{Li}^{+} @ \mathrm{C}_{60}$, the final state of the $\mathrm{ET}$ reaction, [10] $\mathrm{CPP}^{+}$has a smaller solvation energy as compared to the initial state $\mathrm{Li}^{+} @ \mathrm{C}_{60}$, which results in a hypsochromic shift of the CT band (positive $\Delta h v$ in eq. (1))

Electron transfer rates. Encapsulation of $\mathrm{Li}^{+}$by the fullerene is also associated by modulating the PET rate. The non-adiabatic electron transfer rate, $\mathrm{k}_{\mathrm{ET}}$, was estimated using the classical Marcus equation ${ }^{[22]}$ (for details see the Supporting Information). The computed values of ET parameters (the Gibbs energy $\Delta G_{0}$, electronic coupling $\mathrm{V}$, and the reorganization energy $\lambda$ ) and $\mathrm{K}_{\mathrm{ET}}$ for CS in $\mathrm{C}_{60} \subset[10] \mathrm{CPP}$ and $\mathrm{Li}^{+} @ \mathrm{C}_{60} \subset[10] \mathrm{CPP}$ in the polar (benzonitrile and dichloromethane), and nonpolar (diisopropyl ether) medium are listed in Table 2.

The PET in $\mathrm{C}_{60} \subset[10] \mathrm{CPP}$ occurs in normal Marcus regime $\left(\left|\Delta \mathrm{G}_{0}\right|\right.$ $<\lambda$ ) on the nanosecond time scale, $\mathrm{k}_{\mathrm{ET}} \approx 1.1$ and $1.7 \mathrm{~ns}^{-1}$ in nonpolar and polar solvents, respectively. As expected, $\Delta G_{0}$ decreases as the solvent polarity increases. 
Table 2. ET parameters and the rate for charge separation reactions of $\mathrm{C}_{60} \subset[10] \mathrm{CPP}$ and $\mathrm{Li}^{+} @ \mathrm{C}_{60} \subset[10] \mathrm{CPP}$ complexes in diisopropyl ether (DIPE), dichloromethane (DCM), and benzonitrile (BZN).

\begin{tabular}{lccccc}
\hline Complex & Solv. & $\Delta \mathbf{G}_{0}{ }^{\mathbf{a}}, \mathbf{e V}$ & $|\mathbf{V}|, \mathbf{e V}$ & $\boldsymbol{\lambda}, \mathbf{e V}$ & $\mathbf{k E T}_{\mathrm{ET}} \mathbf{s}^{-1}$ \\
\hline $\mathrm{C}_{60} \subset[10] \mathrm{CPP}$ & $\mathrm{DIPE}$ & 0.111 & 0.0021 & 0.233 & $1.08 \cdot 10^{9}$ \\
& $\mathrm{DCM}$ & 0.066 & 0.0021 & 0.330 & $1.24 \cdot 10^{9}$ \\
& $\mathrm{BZN}$ & 0.049 & 0.0021 & 0.338 & $1.68 \cdot 10^{9}$ \\
$\mathrm{Li}^{+} @ \mathrm{C}_{60} \subset[10] \mathrm{CPP}$ & $\mathrm{DIPE}$ & -0.576 & 0.0024 & 0.232 & $1.39 \cdot 10^{9}$ \\
& $\mathrm{DCM}$ & -0.464 & 0.0024 & 0.315 & $8.61 \cdot 10^{10}$ \\
& $\mathrm{BZN}$ & -0.406 & 0.0024 & 0.322 & $1.37 \cdot 10^{11}$ \\
\hline
\end{tabular}

[a] Gibbs energy difference between LE1 and CS states in solvent.

By contrast, CS in $\mathrm{Li}^{+} @ \mathrm{C}_{60} \subset[10] \mathrm{CPP}$ complex takes place in inverted Marcus region $\left(\left|\Delta G_{0}\right|>\lambda\right)$, and the ET reaction becomes higher with decreasing exergonicity. The rate undergoes a rise from $1.4 \mathrm{~ns}^{-1}$ to $137 \mathrm{~ns}^{-1}$. Note that the rate of charge recombination cannot be accurately estimated since this ET process is found to be in the deep inverted Marcus region $\left(\left|\Delta \mathrm{G}_{0}\right|>>\lambda\right)$.

In conclusion, photoinduced charge separation in the $\mathrm{Li}^{+} @ \mathrm{C}_{60} \subset[10] \mathrm{CPP}$ and $\mathrm{C}_{60} \subset[10] \mathrm{CPP}$ complexes has been studied in detail using the TD-DFT approach. The anomalous solvent effect-destabilization of charge separated states by polar medium has been found for $\mathrm{Li}^{+} @ \mathrm{C}_{60} \subset[10] \mathrm{CPP}$. This is the first example of fullerene based systems where a hypsochromic shift of the CS band is observed. The CS reaction in the $\mathrm{Li}^{+} @ \mathrm{C}_{60} \subset[10] \mathrm{CPP}$ complex is found to occur in the inverted Marcus regime. In spite of that the CS reaction rate becomes faster with increasing solvent polarity of the environment.

\section{Experimental Section}

Full computational Details are given in the supporting information.

\section{Acknowledgements}

We are grateful for financial support from the Spanish MINECO (Network CTQ2016-81911-REDT, project CTQ2017-85341-P, and Juan de la Cierva formación contracts FJCl-2016-29448 to A.J.S. and FJCl-2017-32757 to O.A.S.), and the Catalan DIUE (2017SGR39, XRQTC, and ICREA Academia 2014 Award to M.S.).

Keywords: Photoinduced electron transfer • cycloparaphenylenes $\cdot$ solvent effect $\bullet$ hypsochromic shift • endohedral metallofullerene.

[1] a) G. Bottari, J. A. Suanzes, O. Trukhina, T. Torres, J. Phys. Chem. Lett 2011, 2, 905-913; b) R. L. House, N. Y. M. Iha, R. L. Coppo, L. Alibabaei, B. D. Sherman, P. Kang, M. K. Brennaman, P. G. Hoertz, T. J. Meyer, J Photochem. Photobiol., C 2015, 25, 32-45; c) M. R. Wasielewski, Chem. Rev. 1992, 92, 435-461.
[2] a) F. D'Souza, R. Chitta, K. Ohkubo, M. Tasior, N. K. Subbaiyan, M. E. Zandler, M. K. Rogacki, D. T. Gryko, S. Fukuzumi, J. Am. Chem. Soc. 2008, 130, 14263-14272; b) F. D'Souza, O. Ito, Chem. Soc. Rev. 2012, 41, 86-96; c) S. Fukuzumi, T. Honda, K. Ohkubo, T. Kojima, Dalton Trans. 2009, 3880-3889; d) S. Fukuzumi, K. Ohkubo, T. Suenobu, Acc. Chem. Res. 2014, 47, 1455-1464

[3] a) H. Imahori, D. M. Guldi, K. Tamaki, Y. Yoshida, C. Luo, Y. Sakata, S. Fukuzumi, J. Am. Chem. Soc. 2001, 123, 6617-6628; b) T. Polívka, V Sundström, Chem. Rev. 2004, 104, 2021-2072; c) H. B. Gray, J. R. Winkler, Q. Rev. Biophys. 2004, 36, 341-372.

[4] a) Y. Rio, W. Seitz, A. Gouloumis, P. Vázquez, J. L. Sessler, D. M. Guldi, T. Torres, Chem. - Eur. J. 2010, 16, 1929-1940; b) N. J. L. K. Davis, R W. MacQueen, S. T. E. Jones, C. Orofino-Pena, D. Cortizo-Lacalle, R. G. D. Taylor, D. Credgington, P. J. Skabara, N. C. Greenham, J. Mater. Chem. C 2017, 5, 1952-1962; c) L. Li, J. Han, B. Nguyen, K. Burgess, J. Org. Chem. 2008, 73, 1963-1970; d) V. Leen, D. Miscoria, S. Yin, A. Filarowski, J. Molisho Ngongo, M. Van der Auweraer, N. Boens, W. Dehaen, J. Org. Chem. 2011, 76, 8168-8176.

[5] a) B. Liu, H. Fang, X. Li, W. Cai, L. Bao, M. Rudolf, F. Plass, L. Fan, X. Lu, D. M. Guldi, Chem. - Eur. J. 2015, 21, 746-752; b) G. Rotas, G. Charalambidis, L. Glätzl, D. T. Gryko, A. Kahnt, A. G. Coutsolelos, N. Tagmatarchis, Chem. Commun. 2013, 49, 9128-9130; c) J. L. Sessler, D. Seidel, Angew. Chem. Int. Ed. 2003, 42, 5134-5175.

[6] a) S. Bosi, T. Da Ros, G. Spalluto, M. Prato, Eur. J. Med. Chem. 2003 38, 913-923; b) D. M. Guldi, B. M. Illescas, C. M. Atienza, M. Wielopolski N. Martín, Chem. Soc. Rev. 2009, 38, 1587-1597; c) D. M. Guldi, M. Prato, Acc. Chem. Res. 2000, 33, 695-703; d) M. Izquierdo, B. Platzer A. J. Stasyuk, O. A. Stasyuk, A. A. Voityuk, S. Cuesta, M. Solà, D. M. Guldi, N. Martín, Angew. Chem. Int. Ed. 2019, 131, 7006-7011; e) N. Martín, Chem. Commun. 2006, 2093-2104; f) C. M. Sayes, J. D. Fortner, W. Guo, D. Lyon, A. M. Boyd, K. D. Ausman, Y. J. Tao, B. Sitharaman, L. J. Wilson, J. B. Hughes, J. L. West, V. L. Colvin, Nano Lett. 2004, 4, 1881-1887; g) M. C. Scharber, D. Mühlbacher, M. Koppe, P. Denk, C. Waldauf, A. J. Heeger, C. J. Brabec, Adv. Mater. 2006, 18, 789-794; h) A. J. Stasyuk, O. A. Stasyuk, S. Filippone, N. Martin, M. Solà, A. A Voityuk, Chem. - Eur. J. 2018, 24, 13020-13025; i) A. J. Stasyuk, O. A. Stasyuk, M. Solà, A. A. Voityuk, Chem. - Eur. J. 2019, 25, 2577-2585; j) M. Barrejón, A. Mateo-Alonso, M. Prato Eur. J. Org. Chem. 2019, 33713383.

[7] Y. Araki, O. Ito, J. Photochem. Photobiol., C 2008, 9, 93-110.

[8] a) G. Bottari, G. de la Torre, D. M. Guldi, T. Torres, Chem. Rev. 2010 110, 6768-6816; b) M. D. Ward, Chem. Soc. Rev. 1997, 26, 365-375; c) T. Hayashi, H. Ogoshi, Chem. Soc. Rev. 1997, 26, 355-364. D) Q. Huang, G. Zhuang, H. Jia, M. Qian, S. Cui, S. Yang, P. Du, Angew. Chem. Int. Ed. 2019, 58, 6244-6249.

[9] a) J. L. Atwood, G. A. Koutsantonis, C. L. Raston, Nature 1994, 368, 229 231; b) T. Haino, M. Yanase, Y. Fukazawa, Angew. Chem. Int. Ed. 1997, 36, 259-260; c) P. D. W. Boyd, C. A. Reed, Acc. Chem. Res. 2005, 38, 235-242; d) K. Tashiro, T. Aida, Chem. Soc. Rev. 2007, 36, 189-197; e) E. M. Pérez, L. Sánchez, G. Fernández, N. Martín, J. Am. Chem. Soc. 2006, 128, 7172-7173; f) J. L. Segura, N. Martín, Angew. Chem. Int. Ed. 2001, 40, 1372-1409; g) E. M. Pérez, N. Martín, Chem. Soc. Rev. 2008, $37,1512-1519$.

[10] a) S. Mizyed, P. E. Georghiou, M. Bancu, B. Cuadra, A. K. Rai, P. Cheng, L. T. Scott, J. Am. Chem. Soc. 2001, 123, 12770-12774; b) T. Kawase, K. Tanaka, N. Fujiwara, H. R. Darabi, M. Oda, Angew. Chem. Int. Ed. 2003, 42, 1624-1628; c) T. Kawase, M. Oda, in Pure Appl. Chem. 2006, 78, 831-841.

[11] a) P. J. Evans, E. R. Darzi, R. Jasti, Nat. Chem. 2014, 6, 404; b) M. P. Alvarez, P. M. Burrezo, M. Kertesz, T. Iwamoto, S. Yamago, J. Xia, R. Jasti, J. T. L. Navarrete, M. Taravillo, V. G. Baonza, J. Casado, Angew. Chem. Int. Ed. 2014, 53, 7033-7037; c) H. Omachi, Y. Segawa, K. Itami, Acc. Chem. Res. 2012, 45, 1378-1389; d) E. Kayahara, V. K. Patel, S. Yamago, J. Am. Chem. Soc. 2014, 136, 2284-2287; e) M. Fujitsuka, D. W. Cho, T. Iwamoto, S. Yamago, T. Majima, Phys. Chem. Chem. Phys. 
2012, 14, 14585-14588; f) E. Kayahara, T. Kouyama, T. Kato, S. Yamago, J. Am. Chem. Soc. 2016, 138, 338-344.

[12] a) T. Kawase, H. Kurata, Chem. Rev. 2006, 106, 5250-5273; b) Y. Nakanishi, H. Omachi, S. Matsuura, Y. Miyata, R. Kitaura, Y. Segawa, K. Itami, H. Shinohara, Angew. Chem. Int. Ed. 2014, 53, 3102-3106; c) J. Xia, J. W. Bacon, R. Jasti, Chem. Sci. 2012, 3, 3018-3021; d) T. Iwamoto, Y. Watanabe, T. Sadahiro, T. Haino, S. Yamago, Angew. Chem. Int. Ed. 2011, 50, 8342-8344; e) T. Iwamoto, Z. Slanina, N. Mizorogi, J. Guo, T. Akasaka, S. Nagase, H. Takaya, N. Yasuda, T. Kato, S. Yamago, Chem - Eur. J. 2014, 20, 14403-14409.

[13] H. Ueno, T. Nishihara, Y. Segawa, K. Itami, Angew. Chem. Int. Ed. 2015, 54, 3707-3711.

[14] a) A. D. Becke, Phys. Rev. A 1988, 38, 3098-3100; b) C. Lee, W. Yang, R. G. Parr, Phys. Rev. B 1988, 37, 785-789; c) F. Weigend, R. Ahlrichs, Phys. Chem. Chem. Phys. 2005, 7, 3297-3305; d) F. Weigend, Phys. Chem. Chem. Phys. 2006, 8, 1057-1065.
[15] T. Yanai, D. P. Tew, N. C. Handy, Chem. Phys. Lett. 2004, 393, 51-57.

[16] I. González-Veloso, J. Rodríguez-Otero, E. M. Cabaleiro-Lago, Phys Chem. Chem. Phys. 2016, 18, 31670-31679.

[17] K. Yuan, C.-H. Zhou, Y.-C. Zhu, X. Zhao, Phys. Chem. Chem. Phys 2015, 17, 18802-18812.

[18] a) M. A. Hashmi, M. Lein, J. Phys. Chem. C 2018, 122, 2422-2431; b) A. J. Stasyuk, O. A. Stasyuk, M. Solà, A. A. Voityuk, J. Phys. Chem. C 2019 DOI: $10.1021 /$ acs.jpcc.9b02354.

[19] J. Tomasi, B. Mennucci, R. Cammi, Chem. Rev. 2005, 105, 2999-3094.

[20] a) B. Bagchi, Annu. Rev. Phys. Chem. 1989, 40, 115-141; b) G. J. Kavarnos, N. J. Turro, Chem. Rev. 1986, 86, 401-449.

[21] B. Mennucci, Wiley Interdiscip. Rev.: Comput. Mol. Sci. 2012, 2, 386-404.

[22] R. A. Marcus, N. Sutin, Biochim. Biophys. Acta, Rev. Bioenerg. 1985 $811,265-322$ 
Entry for the Table of Contents (Please choose one layout)

Layout 1:

\section{COMMUNICATION}

Blue shift of charge separated states generated by photoexcitation of $\mathrm{Li}^{+} @ \mathrm{C}_{60} \subset[10] \mathrm{CPP}$ complexes in the polar medium.

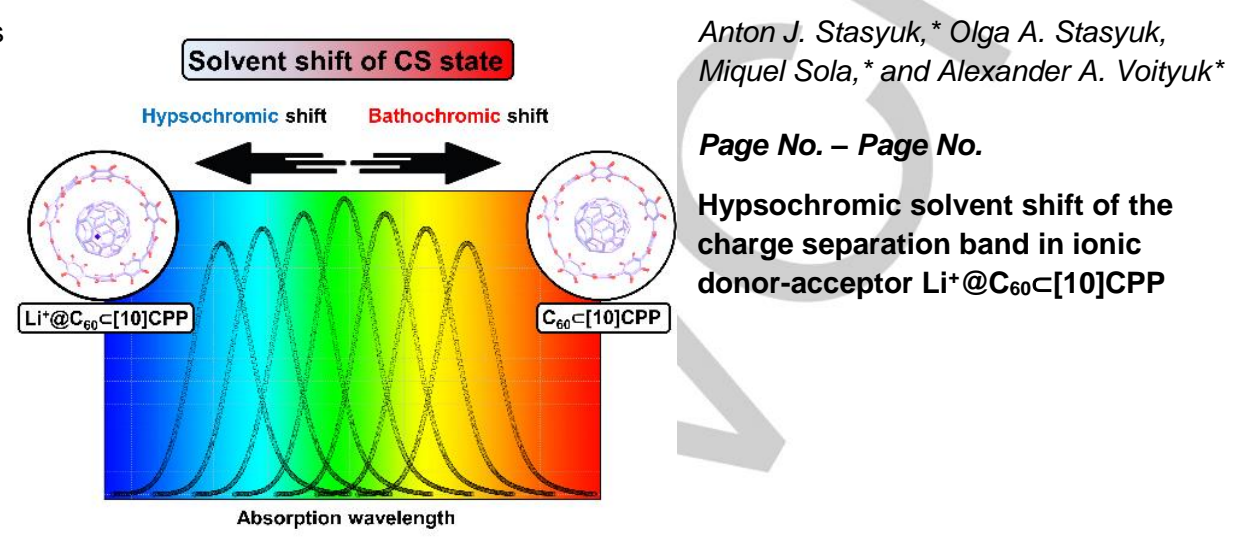

Anton J. Stasyuk, ${ }^{*}$ Olga A. Stasyuk, Miquel Sola, ${ }^{*}$ and Alexander A. Voityuk

Page No. - Page No.

Hypsochromic solvent shift of the charge separation band in ionic donor-acceptor $\mathrm{Li}^{+} @ \mathrm{C}_{60} \subset[10] \mathrm{CPP}$ 